\title{
Are Psychedelics Something New in Teaching Psychopharmacology?
}

\author{
Alan K. Louie ${ }^{1} \cdot$ Eugene V. Beresin $^{2} \cdot$ John Coverdale ${ }^{3} \cdot$ Richard Balon $^{4} \cdot$ Anthony P. S. Guerrero $^{5} \cdot$ Mary K. Morreale $^{4}$. \\ Rashi Aggarwal $^{6} \cdot$ Adam M. Brenner $^{7}$
}

Published online: 28 August 2020

(C) Academic Psychiatry 2020

These are interesting times for the practice and teaching of psychopharmacology. For instance, the repurposing of ketamine, the dissociative anesthetic, from anesthesiology into the practice of psychiatry, has garnered considerable interest as a potential innovation in the treatment of major depression [1]. Similarly attracting attention is the re-emergence of psychedelic drugs as potential treatments for a wide range of psychiatric disorders [2, 3]. Baby-boomer psychiatrists will remember the age of psychedelics in the 1960s [4], while subsequently trained generations of psychiatrists rarely, if ever, heard about them as potential treatments. A hiatus occurred for psychedelic research, from the mid-1970s into the mid-1990s [4]. Despite this hiatus, careful scientific studies with psychedelics began in the mid1990s, by a few investigators [2]. A July 31, 2020, ClinicalTrials.gov search using the term psychedelics [5] listed 268 National Institute of Health trials for the treatment of both medical and psychiatric conditions, the latter of which include depression, anxiety, obsessive compulsive disorder, posttraumatic stress disorder (PTSD), substance use disorders, and cognitive impairment.

Psychedelic drugs $[2,3]$ include "classical" psychedelics like lysergic acid diethylamide (LSD), psilocybin, and ayahuasca, which are agonists at the serotonin $2 \mathrm{~A}$ receptor, and empathogens like 3,4-methylenedioxymethamphetamine (MDMA), which release and inhibit the reuptake of serotonin

Alan K. Louie

Louiemd@stanford.edu

\footnotetext{
Stanford University, Stanford, CA, USA

Harvard Medical School, Boston, MA, USA

Baylor College of Medicine, Houston, TX, USA

Wayne State University, Detroit, MI, USA

5 University of Hawai'i John A. Burns School of Medicine, Honolulu, HI, USA

6 Rutgers New Jersey Medical School, Newark, NJ, USA

7 University of Texas Southwestern Medical Center, Dallas, TX, USA
}

and dopamine. Sometimes, other compounds, like ketamine, and atypical hallucinogens are also called psychedelics. Psilocybin, found in some mushrooms, and ayahuasca, consisting of two plant-based compounds, have been part of sacred ceremonies of ancient cultures and provide an interface between Western psychiatry and traditional indigenous healing approaches.

State-of-the-art human studies with psychedelics are not easy to conduct given regulatory challenges and complex design issues, including providing an appropriate control group and blinding. Nevertheless, over the past couple decades, enough evidence has gradually accumulated to warrant Food and Drug Administration (FDA) trials. Psilocybin is currently in early phase 2 trials for the treatment of major depressive disorder [6] and treatment-resistant depression [7] under an FDA breakthrough therapy designation. MDMA is in the midst of phase 3 trials for PTSD [8], also under a breakthrough therapy designation. Time will tell whether these treatments will ultimately gain FDA approval, but their reemergence may represent a teachable moment.

This editorial focuses on teaching residents about these fascinating and ancient compounds called psychedelics, because they present an opportunity to educate our residents regarding aspects of the scientific method, including its relationship to the social, historical, political, and cultural factors that influence research, the clinical response to public experimentation, and policy. We have parsed the teachable moments into basic, practical, and curious lessons from psychedelics.

\section{Basic Lessons}

Trainees may ask, "If some people now claim that psychedelics can ameliorate psychiatric symptoms, why wasn't it noted previously?" Although we should defer to medical historians to thoroughly sort this out, at least two points come to mind. First, potential benefits were suspected, and approximately 1000 studies were said to have been conducted back 
in the 1960s [4]. These studies, however, were conducted before scientifically rigorous clinical trial methodologies were implemented. The lesson is that even drugs with significant effect sizes need to be studied under rigorous conditions to prove their efficacy statistically. Common problems stemmed from the lack of control groups, randomization of subjects, objective outcome measures, blinding, establishment of dose-response relationships, standardization of treatment conditions in concert with drug administration (e.g., use of manualized psychotherapy during administration), precise definition of the symptoms or disorders being treated [9], evaluation of the longevity of response, and more.

A second point is that studies on psychedelics were for the most part shut down in the 1970s. Legal restrictions (e.g., designation of LSD as a schedule 1 drug) on psychedelic drug research made studies so difficult to conduct that a hiatus occurred [4]. The hiatus grew out of public opinion, which had turned against psychedelics following relatively uncontrolled availability of these agents, and multiple press reports of adverse outcomes from use of psychedelics. For instance, media coverage of frank misuse of psychedelics at the Woodstock music festival to reports of psychedelics causing schizophrenia gave researchers pause from pursuing further the potential positive use of these substances. Thereafter, any claims of therapeutic benefit were eclipsed by descriptions of fear-provoking, psychotic-like reactions ("bad trips") on psychedelics. Stigmatization of psychedelics and research on them followed and persists today. Rigorous studies of the behavioral pharmacology of psychedelics only began to reappear slowly and accumulate over the last 25 years, and the field is still in its infancy [2].

Thus, the lesson here for trainees is part scientific and part sociological. First, rigorous scientific studies and clinical trials (both quantitative and qualitative) are labor intensive, time consuming, and expensive. They are indispensable, nevertheless, to make sense of and sort out the naturalistic reports of psychedelic effects (both short term and long term). Second, psychiatrists need to be part of not only the scientific work but also advocacy for rational drug policies based on science, without glorification or demonization of psychedelics. The reported risks and benefits need to be anchored in evidence, described objectively, and devoid of politics and commercial interests. Despite the public's current desperation for new and effective treatments, researchers must look for clinically significant drug effects under controlled conditions for specific indications [9], evaluate the conditions for safe and ethical administration, and define exclusion criteria. Psychiatrists must protect those at risk for possible side effects of psychedelics, including those with psychotic spectrum disorders and misuse histories. And psychiatrists must advocate for rational policymaking, as US society navigates the issues of diversion, microdosing for cognitive enhancement, and commercialization of psychedelics. The history lesson from the 1960s is that any promise, now being investigated, of psychedelics treating psychiatric disorders could easily go sideways if we do not proceed with caution and a "do no harm" perspective.

\section{Practical Lessons}

What should residents say if a patient asks them about the use of psychedelics for their psychiatric symptoms? Of course, the first response is that treatment should depend on scientific evidence. Moreover, psilocybin is an example of a psychedelic compound that is a schedule 1 drug and not currently FDA approved and therefore cannot be prescribed. It should also be appreciated that patients may be seeking advice before trying to obtain psychedelics through underground distribution networks. Public interest has been stimulated by a wide range of lay or journalistic sources, including the bestselling book by Michael Pollan about psychedelics [10]. Positive claims in the media and news without evidence are not to be trusted. As new medications are approved by the FDA and brought to market, psychiatrists have long been taught to objectively review the prescribing information and other available data and cautiously decide on when to introduce medications into their practices. Currently, such review and decision-making processes cannot be done with psychedelics, because the clinical trials are ongoing, and the full extent of risks, benefits, and utility for various disorders are unknown. In sum, some patients are now voicing interest in trying psychedelics well before psychiatrists have the data needed to provide scientific information about psychedelics as treatments.

Faculty must help residents to address this knowledge gap as best as possible with information about clinical trials that have occurred since the 1990s, as well as ongoing trials for FDA approval. Given public interest and underground availability, residents should learn how to respond to patients and explore their queries. It is not unlike responding to patients who ask about the therapeutic effects of tetrahydrocannabinol (THC) or cannabidiol (CBD), when not enough rigorous scientific research is available to make informed decisions. Though accurate, a terse answer, like "It is not FDA approved to treat your disorder," would run the risk of sending the message that the resident is not willing to discuss the patient's questions, perhaps due to stigmatized views, fear of legal ramifications, lack of knowledge, or other concerns. An undesirable outcome would be that patients would get the idea that the use of psychedelics is outside the treatment dialog and would start self-medicating without telling their psychiatrist.

\section{Curious Lessons}

Trainees may ask, "What about the historical accounts from the 1960s of 'bad trips' with psychedelics?" Investigators of 
drugs like psilocybin have noted that the effects of these drugs have been substantially influenced by the setting in which they were administered. In general, the assumption was that supportive and safe settings lead to beneficial experiences while stressful and uncertain environments might result in "bad" trips. In the past, recreational and global religious/spiritual use has frequently involved group settings, which have varied by level of support. Equally important may be the mindset of the person taking the drug and the person's expectations of impact ("expectancy"). These two factors were coined in the 1960 s as "set and setting" [11,12].

Trainees should be aware that during modern clinical trials with these drugs, investigators have been exceedingly diligent in controlling the set and setting as much as possible to ensure consistency of their influences on drug effects and safety. In typical protocols [12], subjects are continuously monitored and cared for by two trained researchers, a man and a woman, during their 8-hour drug experience in a standardized, bedroom-like laboratory. The next morning subjects return for a session to discuss with researchers the drug experience and its integration with their life. This whole process had been termed "psychedelic-assisted psychotherapy" [8]. Trainees should know that any FDA approval of psychedelics will likely require that psychedelics be administered with similar care and oversight and with investment in trained personnel, instead of merely sending an outpatient home with a new prescription. If a psychedelic is FDA approved, a Risk Evaluation and Mitigation Strategy (REMS) protocol is anticipated. We hope that trainees might be curious about the idea of psychedelic-assisted psychotherapy and recognize that expectations and interactions between the patient and provider during the drug experience might greatly modify the effects of the drug.

Another possible claim is that psychedelic exposure may, under some conditions, have unusually long-term effects. In other words, even after the drug is subsequently cleared from the system, some change in behavior may persist. Some studies described potential beneficial effects of psychedelics, after perhaps only one or two administrations without additional dosing, lasting for days, weeks, or months. This finding contrasts with most psychiatric drugs, which appear to exert effect in a relatively transient manner. For instance, patients with life-threatening cancer had clinically significant decreases in depression and anxiety after only two psilocybin sessions, which, in $80 \%$ of them, persisted at a 6-month follow-up [13]. If such long-term effects are replicated, these findings may support an emerging paradigm in which a psychopharmacological agent enhances long-lasting learning. For instance, when cycloserine was administered with exposure therapy, it was said to have augmented learning from exposure to an anxiety-provoking stimulus, purportedly through the Nmethyl-D-aspartate (NMDA) receptor [14]. A similar paradigm is now being applied to the use of transcranial magnetic stimulation (TMS), in which stimulation of the medial prefrontal cortex, combined with extinction of conditioned fear, accelerated extinction learning [15]. The model is that the planned confluence of a drug (or TMS) with a behavioral intervention, like extinction, will augment relatively lasting change or learning in the brain, greater than that seen with either the drug or behavioral intervention alone.

One could hypothesize that psychedelics may alter behavior in a long-term manner due to new learning enhanced by the drug experience. Functional magnetic resonance imaging (fMRI) studies of subjects under the influence of psilocybin may suggest a mechanism by which the interconnectivities between different regions of the brain were altered [16], perhaps increasing the probability of novel connections being laid down, and thus neuroplasticity and learning [3]. The previously discussed set and setting, guided by psychedelicassisted psychotherapy, might play a critical role in determining which connections are laid down and which are not during the drug experiences.

One last curiosity about psychedelics is that some people report feelings and perceptions beyond their prior comprehension, which they say are indescribable to others, thus having an "ineffable" quality [10]. Perhaps residents will be fascinated with the question of the relationship between the psychedelic state and other altered states of consciousness, induced by meditation, mindfulness, or hypnosis. All of these states have been said to cause lasting behavioral changes, from lowering of blood pressure to smoking cessation, well beyond the period of the treatment. Will researchers be able to differentiate the fMRI images of subjects under the influence of psychedelics and these other states of mind? Functional MRI studies suggested that psychedelics had specific effects on the brain, including some decreases in activity which correlated with subjects reporting "ego dissolution" [17]. The scientific substantiation of these types of claims and comparison to other altered states of consciousness will be a challenge; yet perhaps someday, one of our learners will pursue this study.

\section{Conclusions}

We do not yet know if psilocybin and other psychedelics will survive randomized controlled trials, become FDA-approved, and join the psychopharmacological armamentarium. Nevertheless, we suggest discussing the current and potential impact of the re-emergence of psychedelics on our culture. Basic lessons and an appreciation of history emphasize the importance of scientific rigor and ethics in research methods, the assessment of short- and long-term consequences, and the socioeconomic-political variables that bear upon funding and implementation of protocols. Research of any kind occurs in a historical background and sociocultural climate that influence scientific inquiry. Practical lessons address what to tell 
patients who ask about self-medicating their symptoms with psychedelics, which are already available illicitly. Curious lessons include investigating unusual psychedelic qualitieseffects dependent on set and setting, the potential of long-term effects, and other drug reactions that may be hard to define in words or measure. Perhaps, the most important lesson for psychiatric residents is the need for them to advocate for a scientific determination of the clinical utility of psychedelic drugs and of any policies governing them. In the tradition of "do no harm," psychiatry's first priority should be the safety and ethics of pursuing treatments using psychedelic drugs.

\section{Compliance with ethical standards}

Disclosure On behalf of all authors, the corresponding author states that there is no conflict of interest.

\section{References}

1. Sanacora G, Frye MA, McDonald W, Mathew SJ, Turner MS, Schatzberg AF, et al. A consensus statement on the use of ketamine in the treatment of mood disorders. JAMA Psychiatry. 2017;74: 399-405.

2. Reiff CM, Richman EE, Nemeroff CB, Carpenter LL, Widge AS, Rodriguez CI, et al. Psychedelics and psychedelic-assisted psychotherapy. Am J Psychiatry. 2020;177:391-410.

3. Vollenweider FX, Kometer M. The neurobiology of psychedelic drugs: implications for the treatment of mood disorders. Nat Rev Neurosci. 2010;11:642-51.

4. Belouin SJ, Henningfield JE. Psychedelics: where we are now, why we got here, what we must do. Neuropharmacology. 2018;142:719.

5. National Institutions of Health, U.S. National Library of Medicine. ClinicaTrials.gov. Available at https://clinicaltrials.gov/ct2/results? cond $=\&$ term $=$ psychedelic $\&$ cntry $=\&$ state $=\&$ city $=\&$ dist $=$ Accessed 10 May 2020.

6. National Institutions of Health, U.S. National Library of Medicine, ClinicalTrials.gov. A study of psilocybin for major depressive disorder (MDD). Available at https://clinicaltrials.gov/ct2/show/ NCT03866174?term $=$ psilocybin\&intr=Psilocybin\&sfpd_s $=07 \%$ 2 F30\%2F2018\&sfpd_e $=03 \% 2$ F21\%2F2020\&draw $=2 \&$ rank $=1$. Accessed 31 July 2020.
7. National Institutions of Health, U.S. National Library of Medicine, ClinicalTrials.gov. The safety and efficacy of psilocybin in participants with treatment resistant depression (P-TRD). Available at https://clinicaltrials.gov/ct2/show/NCT03775200? term=psilocybin\&recrs=abd\&draw=3. Accessed 31 July 2020.

8. Mithoefer MC, Feduccia AA, Jerome L, Mithoefer A, Wagner M, Walsh Z, et al. MDMA-assisted psychotherapy for treatment of PTSD: study design and rationale for phase 3 trials based on pooled analysis of six phase 2 randomized controlled trial. Psychopharmacology. 2019;236(9):2735-45. https://doi.org/10. 1007/s00213-019-05249-5.

9. Schatzberg AF. Some comments on psychedelic research. Am J Psychiatry. 2020;177:368-9.

10. Pollan M. How to change your mind: what the new science of psychedelics teaches us about consciousness, dying, addiction, depression, and transcendence. London: Penguin Books; 2018.

11. Carhart-Harris RL, Roseman L, Haijen E, Erritzoe D, Watts R, Branchi I, et al. Psychedelics and the essential importance of context. J Psychopharmacol. 2018;32:725-31.

12. Garcia-Romeu A, Richards WA. Current perspectives on psychedelic therapy: use of serotonergic hallucinogens in clinical interventions. Int Rev Psychiatry. 2018;30:291-316.

13. Griffiths RR, Johnson MW, Carducci MA, Umbricht A, Richards WA, Richards BD, et al. Psilocybin produces substantial and sustained decreases in depression and anxiety in patients with lifethreatening cancer: a randomized double-blind trial. J Psychopharmacol. 2016;30(12):1181-97.

14. Ebrahimi C, Gechter J, Lueken U, Schlagenhauf F, Wittchen HU, Hamm AO, et al. Augmenting extinction learning with Dcycloserine reduces return of fear: a randomized, placebocontrolled fMRI study. Neuropsychopharmacology. 2020;45:499506.

15. Guhn A, Dresler T, Andreatta M, et al. Medial prefrontal cortex stimulation modulates the processing of conditioned fear. Front Behav Neurosci. 2014;8:44.

16. Preller KH, Razi A, Zeidman P, Stämpfli P, Friston KJ, Vollenweider FX. Effective connectivity changes in LSDinduced altered states of consciousness in humans. Proc Natl Acad Sci U S A. 2019;116:2743-8.

17. Carhart-Harris RL, Muthukumaraswamy S, Roseman L, Kaelen M, Droog W, Murphy K, et al. Neural correlates of the LSD experience revealed by multimodal neuroimaging. Proc Natl Acad Sci U S A. 2016;113:4853-8.

Publisher's Note Springer Nature remains neutral with regard to jurisdictional claims in published maps and institutional affiliations. 